\title{
Hydrogen as a Cause of Doping in Zinc Oxide
}

\author{
Chris G. Van de Walle* \\ Fritz-Haber-Institut der Max-Planck-Gesellschaft, Faradayweg 4-6, D-14 195 Berlin-Dahlem, Germany, \\ and Paul-Drude-Institut, Hausvogteiplatz 5-7, D-10117 Berlin, Germany
}

(Received 3 February 2000)

\begin{abstract}
Zinc oxide, a wide-band-gap semiconductor with many technological applications, typically exhibits $n$-type conductivity. The cause of this conductivity has been widely debated. A first-principles investigation, based on density functional theory, produces strong evidence that hydrogen acts as a source of conductivity: it can incorporate in high concentrations and behaves as a shallow donor. This behavior is unexpected and very different from hydrogen's role in other semiconductors, in which it acts only as a compensating center and always counteracts the prevailing conductivity. These insights have important consequences for control and utilization of hydrogen in oxides in general.
\end{abstract}

PACS numbers: $61.72 . \mathrm{Bb}, 61.72 . \mathrm{Vv}, 71.55 . \mathrm{Gs}$

Because of its unique piezoelectric, optical, and electrical properties, zinc oxide is widely used for device applications including transducers, phosphors, and varistors. Recent progress in single-crystal growth [1] has also opened up prospects for optoelectronic applications, as a substrate for growth of $\mathrm{GaN}$ or as a light-emitting material in its own right. Zinc oxide almost always exhibits strong $n$-type conductivity, with electrons in the conduction band as the charge carriers. Despite years of investigations, the source of this conductivity is still controversial. Because of its prevalence, the $n$-type conductivity has traditionally been attributed to native defects [2]. A recent firstprinciples investigation [3] revealed, however, that none of the native defects exhibits characteristics consistent with a high-concentration shallow donor. Only vacancies have sufficiently low energies to form during synthesis of the material, but zinc vacancies behave as deep acceptors and oxygen vacancies as deep donors. The prevailing $n$-type conductivity can therefore not be attributed to native defects; it must thus be caused by impurities that are unintentionally incorporated.

In this Letter we show, based on first-principles density functional calculations, that hydrogen is an excellent candidate for such an impurity. Hydrogen is ubiquitous and very difficult to remove from the crystal growth environment. It also forms a strong bond with oxygen, providing a powerful driving force for its incorporation in the $\mathrm{ZnO}$ crystal. This incorporation is accompanied by remarkably large relaxations of the surrounding atoms. The resulting $\mathrm{O}-\mathrm{H}$ bonding unit can, in fact, be regarded as a new type of dopant atom, the addition of the proton turning the oxygen into an element behaving much like fluorine.

The present study also reveals a novel type of behavior for hydrogen as an impurity in semiconducting or insulating materials. In all other semiconductors, interstitial hydrogen has been found (theoretically as well as experimentally) to act as an amphoteric impurity [4]: in $p$-type material, hydrogen incorporates as $\mathrm{H}^{+}$(a donor), and in $n$-type material as $\mathrm{H}^{-}$(an acceptor), always counteracting the prevailing conductivity of the material. This amphoteric behavior precludes hydrogen from acting as a dopant, i.e., from being a source of conductivity. In $\mathrm{ZnO}$, however, we find that hydrogen occurs exclusively in the positive charge state; i.e., it always acts as a donor. This opens up prospects for similar behavior in other oxides, in which hydrogen may be used to actively modify the electrical characteristics.

Our first-principles calculations are based on densityfunctional theory (DFT) within the local density approximation (LDA) [5] and the pseudopotential-plane-wave method [6]. In addition to studying the wurtzite (WZ) structure, which is the stable phase for $\mathrm{ZnO}$, we also investigated the zinc-blende (ZB) phase. The atomic and electronic structures of interstitial hydrogen, as well as the energetics, were found to be very similar in these two phases. The impurity studies were carried out in supercells containing 96 atoms for the WZ structure and 64 atoms for the ZB structure, using 2 or 3 special $k$ points in the irreducible part of the Brillouin zone. Effects of the $\mathrm{Zn} 3 d$ states were included using the nonlinear core correction (nlcc) [7], with an energy cutoff of 40 Ry. Test calculations with explicit inclusion of the $\mathrm{Zn} 3 d$ electrons as valence electrons (with a 70 Ry cutoff) confirmed the validity of the conclusions obtained with the nlcc. Structural optimizations for bulk $\mathrm{ZnO}$ in the $\mathrm{WZ}$ structure produced the following parameters: $a=3.241 \AA$, $c / a=1.614$, and $u=0.378$, in satisfactory agreement with experiment $(a=3.25 \AA, c / a=1.602$, and $u=0.382$ ). The computed band gap is $1.81 \mathrm{eV}$ for $\mathrm{ZB}$ and $1.91 \mathrm{eV}$ for $\mathrm{WZ}$, smaller than the experimental gap of $3.4 \mathrm{eV}$ [8] due to the well known DFT-LDA band-gap error; we will discuss the band-gap error below.

Formation energies are foremost among the quantities that can be derived from the first-principles calculations. In equilibrium, the formation energy $E^{f}$ determines the concentration $c$ of the impurity in the semiconductor, through the expression

$$
c=N_{\text {sites }} \exp \left(-E^{f} / k T\right),
$$




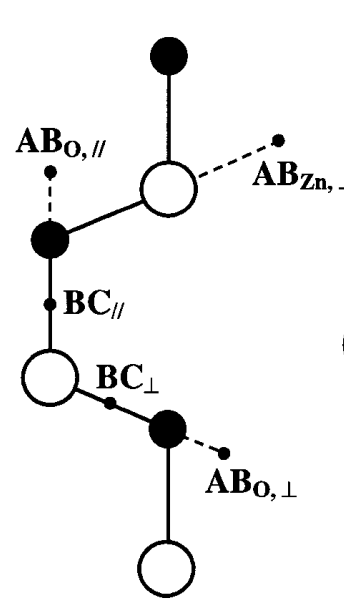

(a)

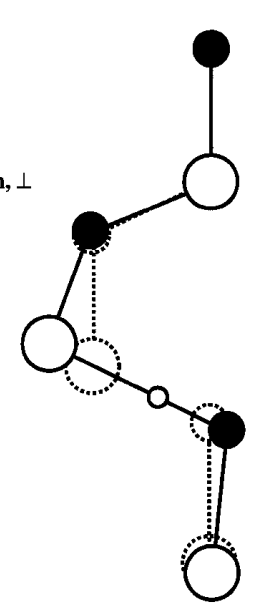

(b)
FIG. 1. (a) Schematic representation of the $\mathrm{ZnO}$ wurtzite structure, and the sites on which interstitial hydrogen can incorporate. $\mathrm{BC}$ indicates the bond-center sites, and $\mathrm{AB}$ indicates the antibonding sites. (b) Relaxed atomic positions of hydrogen and host atoms in the $\mathrm{BC}_{\perp}$ configuration. Ideal lattice positions are shown in dotted lines. Note the magnitude of the relaxations, particularly of the $\mathrm{Zn}$ atom.

where $N_{\text {sites }}$ is the number of sites in the lattice where the impurity can be incorporated, $k$ is Boltzmann's constant, and $T$ is the temperature. The formation energy of a hydrogen interstitial in charge state $q$ is defined as

$E^{f}\left(\mathrm{H}^{q}\right)=E^{\text {tot }}\left(\mathrm{H}^{q}\right)-E^{\text {tot }}($ bulk $)+E_{\mathrm{ZPE}}-\mu_{\mathrm{H}}+q E_{F}$,

where $E^{\text {tot }}\left(\mathrm{H}^{q}\right)$ is the total energy derived from a supercell calculation for the interstitial $\mathrm{H}$ impurity, and $E^{\text {tot }}$ (bulk) is the total energy for a supercell containing only bulk $\mathrm{ZnO}$. $E_{\mathrm{ZPE}}$ is the zero-point energy (ZPE), obtained from the vibrational mode frequencies of the impurity as described below. $\mu_{\mathrm{H}}$ is the hydrogen chemical potential, i.e., the energy of the reservoir with which $\mathrm{H}$ atoms are exchanged.
For presentation purposes, we fix the value of $\mu_{\mathrm{H}}$ to half the energy of an $\mathrm{H}_{2}$ molecule (including the ZPE); our calculated binding energy for $\mathrm{H}_{2}$ (including $\mathrm{ZPE}$ ) is $2.25 \mathrm{eV}$ per atom, within $0.01 \mathrm{eV}$ of the experimental value. More generally, the dependence of $E^{f}$ on $\mu_{\mathrm{H}}$ can be examined by referring to Eq. (2). Finally, $E_{F}$ is the Fermi level, i.e., the chemical potential of the reservoir with which electrons are exhanged. Further details about the formalism can be found in Ref. [9].

For each charge state (which is determined by fixing the number of electrons in the supercell), we have performed extensive investigations to determine stable and metastable positions of the impurity in the lattice. The hydrogen impurity was placed in many different sites in the lattice, and at each location the surrounding host atoms were allowed to fully relax. Sites for which the total energy surface for interstitial hydrogen exhibits a local minimum are depicted in Fig. 1(a). The results are displayed in Table I. For all charge states, the $\mathrm{BC}_{\perp}$ configuration (bond centered in a bond that is not parallel to the $c$ axis) is most stable. An $\mathrm{O}-\mathrm{H}$ bond is formed, pointing along the direction of the original $\mathrm{O}-\mathrm{Zn}$ bond. The corresponding formation energies are shown in Fig. 2.

Table I includes the lowest-energy configurations; for $\mathrm{H}^{+}$, we have also included metastable configurations that are less than $1.0 \mathrm{eV}$ higher in energy. Note that various configurations, in which hydrogen is located at a distance of $\sim 1 \AA$ from the oxygen host atom, are very close in energy. This indicates that the strength of the $\mathrm{O}-\mathrm{H}$ bond is the main driving force for stabilization of this configuration, while details of the hydrogen orientation and relaxations have only small effects on the energy. The size of the lattice relaxations is remarkable; in particular, for the bondcenter (BC) configuration the $\mathrm{Zn}$ atom moves outward over a distance equal to $40 \%$ of the bond length $(0.8 \AA)$, to a position slightly beyond the plane of its nearest neighbors as illustrated in Fig. 1(b). To assist in experimental identification of the hydrogen center, we have calculated the vibrational frequencies of the $\mathrm{O}-\mathrm{H}$ stretching and wagging

TABLE I. Calculated formation energies $E^{f}$ [Eq. (2)] and lattice relaxations for an interstitial hydrogen impurity in wurtzite ZnO, for various lattice locations and charge states. The symbol $\|$ denotes configurations with the O-H bond parallel to the $c$ axis ([0001]); the other bond directions are denoted by the symbol $\perp . E^{f}$ is given for a Fermi level at the top of the valence band, and with $\mu_{\mathrm{H}}$ determined by the energy of the $\mathrm{H}_{2}$ molecule. Zero-point energies are included as described in the text. $\Delta d_{\mathrm{Zn}(\mathrm{O})}$ denotes the displacement of the nearest neighbor $\mathrm{Zn}(\mathrm{O})$ atom from its nominal lattice site, expressed as a percentage of the bond length. $d_{\mathrm{Zn}-\mathrm{H}}$ denotes the $\mathrm{Zn}-\mathrm{H}$ distance, and $d_{\mathrm{O}-\mathrm{H}}$ the $\mathrm{O}-\mathrm{H}$ distance, in $\AA$.

\begin{tabular}{ccccccc}
\hline \hline Charge state & Location & $\begin{array}{c}E^{f} \\
(\mathrm{eV})\end{array}$ & $\Delta d_{\mathrm{Zn}}$ & $\Delta d_{\mathrm{O}}$ & $\begin{array}{c}d_{\mathrm{Zn}-\mathrm{H}} \\
(\AA)\end{array}$ & $\begin{array}{c}d_{\mathrm{O}-\mathrm{H}} \\
(\AA)\end{array}$ \\
\hline $\mathrm{H}^{+}$ & $\mathrm{BC}_{\perp}$ & -1.84 & $40.6 \%$ & $11.2 \%$ & 2.00 & 0.99 \\
$\mathrm{H}^{+}$ & $\mathrm{BC}_{\|}$ & -1.82 & $39.1 \%$ & $11.8 \%$ & 1.98 & 0.99 \\
$\mathrm{H}^{+}$ & $\mathrm{AB}_{\mathrm{O}, \perp}$ & -1.78 & $18.6 \%$ & $20.6 \%$ & & 1.01 \\
$\mathrm{H}^{+}$ & $\mathrm{AB}_{\mathrm{O}, \|}$ & -1.59 & $20.1 \%$ & $17.8 \%$ & & 1.01 \\
$\mathrm{H}^{0}$ & $\mathrm{BC}_{\perp}$ & 1.07 & $39.9 \%$ & $10.4 \%$ & 1.97 & 0.99 \\
$\mathrm{H}^{-}$ & $\mathrm{BC}_{\perp}$ & 3.92 & $40.7 \%$ & $12.0 \%$ & 2.02 & 1.00 \\
$\mathrm{H}_{2}$ & $\mathrm{AB}_{\mathrm{Zn}, \perp}$ & 0.84 & & & & \\
\hline \hline
\end{tabular}




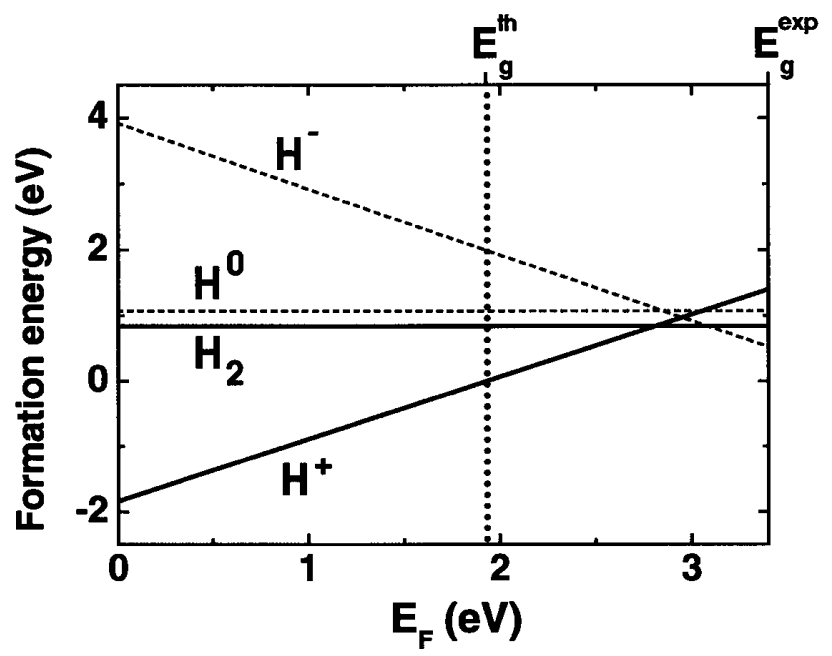

FIG. 2. Formation energies of interstitial hydrogen in $\mathrm{ZnO}$, as a function of Fermi level, obtained from DFT-LDA calculations and referenced to the energy of a free $\mathrm{H}_{2}$ molecule. For each charge state, only the lowest-energy configuration is shown. Zero-point energies are included. The zero of Fermi energy is chosen at the top of the valence band, and both the theoretical $\left(E_{g}^{\text {th }}=1.91 \mathrm{eV}\right.$, dotted line $)$ and experimental $\left(E_{g}^{\exp }=3.4 \mathrm{eV}\right)$ band gaps are indicated. The energies for $\mathrm{H}^{0}$ and $\mathrm{H}^{-}$are shown in dashed lines to indicate they are underestimated in the LDA calculations; after correction, $\mathrm{H}^{+}$is the lowest-energy state throughout the experimental band gap.

modes (in the harmonic approximation); for $\mathrm{H}^{+}$at $\mathrm{BC}$ we find $3680 \mathrm{~cm}^{-1}$ (stretch) and $450 \mathrm{~cm}^{-1}$ (wag), while for $\mathrm{H}^{+}$at $\mathrm{AB}, \mathrm{O}$ we find $3550 \mathrm{~cm}^{-1}$ (stretch) and $900 \mathrm{~cm}^{-1}$ (wag). Not surprisingly, the values for stretching modes are close to those for $\mathrm{H}_{2} \mathrm{O}$ molecules. The calculated vibrational frequencies were used to estimate the zero-point energy contribution to the formation energy, included in Eq. (2).

Figure 2 shows that $\mathrm{H}^{+}$is the lowest energy state over the entire range of Fermi-level positions within the theoretical LDA band gap (indicated with a dotted line in Fig. 2). We will now argue that $\mathrm{H}^{+}$remains the lowest energy state throughout the experimental band gap. Careful inspection of band structures and wave functions indeed indicates that hydrogen does not induce any defect level in the band gap; rather, the lowest conduction bands acquire a certain degree of hydrogen character, indicative of a hydrogen-induced resonance above the conduction-band minimum. Electrons placed in this resonance, of course, relax to the conduction-band minimum, where they can be bound to the donor in a hydrogenic state [10]. Our analysis indicates that all the relevant conduction-band states shift upwards when the LDA band gap is adjusted. This behavior can be deduced from the pressure dependence of the Kohn-Sham states, as well as from a comparison of the nlcc calculations with results that explicitly include the $3 d$ electrons [10]. The formation energies of $\mathrm{H}^{0}$ and $\mathrm{H}^{-}$ shown in Fig. 2 were obtained by placing electrons in the next available unoccupied state - which turns out to be es- sentially the conduction-band minimum. When corrections to the LDA band gap are applied, these formation energies will therefore increase relative to that of $\mathrm{H}^{+}$. The Fermilevel position where the formation energies of $\mathrm{H}^{+}$and $\mathrm{H}^{0}$ are equal (i.e., the donor level) will remain at or above the conduction-band minimum, and similarly for the acceptor level.

We thus conclude that $\mathrm{H}^{+}$is the stable charge state for all Fermi-level positions. This is distinctly different from the situation in other semiconductors, where $\mathrm{H}$ is amphoteric, and always assumes a charge state that counteracts the prevailing conductivity. In $\mathrm{ZnO}$, only $\mathrm{H}^{+}$is stable, and hence hydrogen exclusively acts as a donor. The formation energy of $\mathrm{H}^{+}$is low enough to allow for a large solubility of hydrogen in $n$-type $\mathrm{ZnO}$. The main reason for the difference in behavior compared to other semiconductors is the large strength of the $\mathrm{O}-\mathrm{H}$ bond, which drives the formation energy of $\mathrm{H}^{+}$down. Figure 2 also shows that the formation energy of $\mathrm{H}^{+}$becomes even lower for Fermi-level positions lower in the band gap, i.e., in $p$-type material. Compensation by hydrogen donors is thus an important concern when acceptor doping of $\mathrm{ZnO}$ is attempted.

In addition to isolated interstitials, we have investigated molecular complexes. $\mathrm{H}_{2}$ molecules prefer a location in the interstitial channel, centered on the $\mathrm{AB}_{\mathrm{Zn}, \perp}$ site, and oriented roughly along the $c$ axis. The $\mathrm{H}-\mathrm{H}$ bond length is $0.798 \AA$ (compared with $0.782 \AA$ for the free molecule, at a 40 Ry cutoff). $\mathrm{H}_{2}$ is a neutral complex and its formation energy is also included in Fig. 2. We have also studied complex formation between hydrogen and native defects. A complex consisting of an oxygen vacancy and a hydrogen atom also behaves as a shallow donor. The calculated binding energy, expressed with respect to $\mathrm{H}^{+}$and $V_{\mathrm{O}}^{0}$, is $0.8 \mathrm{eV}$. Oxygen vacancies are low-energy defects [3] and may form in large concentrations. In $n$-type $\mathrm{ZnO}$, these vacancies would be neutral and electrically inactive, but the addition of hydrogen turns them into shallow donors. The hydrogen atom is located close to the center of the vacancy (to within $0.05 \AA$ ); this configuration can thus also be regarded as a substitutional hydrogen impurity located on an oxygen lattice site.

Experimental indications for hydrogen's behavior as a donor in $\mathrm{ZnO}$ were reported in the 1950s [11-13], $\mathrm{ZnO}$ being the first semiconductor in which the properties of hydrogen were systematically studied. Those results, however, went largely unnoticed during the upsurge in research activity on hydrogen in semiconductors that started about 30 years later. Mollwo [11] observed an increase in the conductivity of $\mathrm{ZnO}$ crystals exposed to hydrogen at temperatures above $200{ }^{\circ} \mathrm{C}$. The increase in the conductivity was demonstrated to be due to indiffusion of hydrogen, for which an activation energy of $0.91 \mathrm{eV}$ was measured [11,12]. An increase in conductivity upon exposure to $\mathrm{H}_{2}$ was also observed by Baik et al. [14], and by Kohiki et al. [15] who introduced hydrogen by proton implantation followed by annealing at $200{ }^{\circ} \mathrm{C}$. All of these 
experimental results indicate that introducing hydrogen into $\mathrm{ZnO}$ does not result in a reduction of the conductivity, which is the expected behavior for hydrogen in other semiconductors. Instead, H shows strong behavior as a donor, consistent with the calculations reported here.

We note that hydrogen is present in many of the techniques commonly used for growth of $\mathrm{ZnO}$, such as vaporphase transport [1], hydrothermal growth, or metal-organic chemical vapor deposition (MOCVD). When growth is carried out in air, water vapor acts as a source of hydrogen. Techniques such as laser ablation or sputtering are sometimes intentionally carried out in a hydrogen atmosphere. In addition, $\mathrm{H}_{2} \mathrm{O}$ is always present as a residual gas in any high vacuum system, serving as a source of hydrogen in techniques such as molecular beam epitaxy. It is also possible that hydrogen can penetrate the crystal when exposed to water; dissociative adsorption was observed in molecular dynamics simulations for $\mathrm{H}_{2} \mathrm{O}$ on $\mathrm{MgO}(100)$ [16].

Finally, we mention the ion channeling experiments used by Ohta $e t$ al. to investigate the lattice positions of the host atoms in $\mathrm{ZnO}$ [17]. They found that $\mathrm{Zn}$ atoms have a much greater tendency to displace from the atomic rows along the $\langle 0001\rangle$ direction than oxygen atoms. This is in agreement with our result that for the lowest-energy configuration of $\mathrm{H}^{+}$, i.e., $\mathrm{BC}_{\perp}$, the displacement of the $\mathrm{Zn}$ atoms is much greater than that of the $\mathrm{O}$ atoms [Table $\mathrm{I}$ and Fig. 1(b)]. The concentration of the point defect giving rise to the displacement was estimated to be on the order of $10^{20} \mathrm{~cm}^{-3}$ [17]. These results are consistent with the presence of hydrogen as an unintentional donor, as suggested in the present work.

In summary, we have presented theoretical evidence for the behavior of hydrogen as a shallow donor in $\mathrm{ZnO}$. In contrast to other semiconductors, hydrogen is not amphoteric in $\mathrm{ZnO}$, but occurs exclusively as a donor. It also gives rise to unusually large lattice relaxations. Controlling the conductivity of $\mathrm{ZnO}$ thus requires careful control of hydrogen exposure during and after growth. We suggest that the behavior observed here for hydrogen in $\mathrm{ZnO}$ is likely to occur in other oxide materials as well. Oxides are widely used for electronic and optoelectronic applications, and the role of hydrogen is only starting to be explored. For instance, it has been suggested that metastability of hydrogen plays a role in photorefractive crystals. The fundamental insights provided by the present work should prove useful for studying and controlling hydrogen in similar systems.
Thanks are due to J. McCaldin for stimulating me to investigate $\mathrm{ZnO}$, to $\mathrm{A}$. Kohan and $\mathrm{G}$. Ceder for fruitful interactions during previous studies on $\mathrm{ZnO}$, to J. Neugebauer for helpful discussions, and to the Alexander von Humboldt Foundation.

*Permanent address: Xerox Palo Alto Research Center, 3333 Coyote Hill Road, Palo Alto, CA 94304.

Email address: vandewalle@parc.xerox.com

[1] D. C. Look et al., Solid State Commun. 105, 399 (1998).

[2] D. C. Look, J. W. Hemsky, and J. R. Sizelove, Phys. Rev. Lett. 82, 2552 (1999), and references therein.

[3] A. F. Kohan, G. Ceder, D. Morgan, and C. G. Van de Walle, Phys. Rev. B 61, 15019 (2000).

[4] Hydrogen in Semiconductors, Semiconductors and Semimetals, edited by J. I. Pankove and N. M. Johnson (Academic Press, Boston, 1991), Vol. 34.

[5] P. Hohenberg and W. Kohn, Phys. Rev. 136, B864 (1964); W. Kohn and L. J. Sham, ibid. 140, A1133 (1965).

[6] M. Bockstedte, A. Kley, J. Neugebauer, and M. Scheffler, Comput. Phys. Commun. 107, 187 (1997).

[7] S. G. Louie, S. Froyen, and M. L. Cohen, Phys. Rev. B 26, 1739 (1982).

[8] O. Madelung, M. Schulz, and H. Weiss, Numerical Data and Functional Relationships in Science and Technology (Springer-Verlag, Berlin, 1982), Vol. 17.

[9] J. Neugebauer and C. G. Van de Walle, in Hydrogen in Semiconductors II, edited by N.H. Nickel, Semiconductors and Semimetals, Vol. 61, series editors R. K. Willardson and E. R. Weber (Academic Press, San Diego, 1999), p. 479 .

[10] Since the electrons contributed by $\mathrm{H}^{0}$ and $\mathrm{H}^{-}$occupy the lowest conduction band, one might expect their formation energies to equal that of $\mathrm{H}^{+}$when $E_{F}=E_{g}^{\text {th }}$; the reason their formation energies are somewhat larger is that in a supercell calculation energy contributions result from eigenvalue occupation at special $k$ points, where the conduction-band energy is higher than at $\Gamma$.

[11] E. Mollwo, Z. Phys. 138, 478 (1954).

[12] D. G. Thomas and J. J. Lander, J. Chem. Phys. 25, 1136 (1956).

[13] J. J. Lander, J. Phys. Chem. Solids 3, 87 (1957).

[14] S. J. Baik, J. H. Jang, C. H. Lee, W. Y. Cho, and K. S. Lim, Appl. Phys. Lett. 70, 3516 (1997).

[15] S. Kohiki, M. Nishitani, T. Wada, and T. Hirao, Appl. Phys. Lett. 64, 2876 (1994).

[16] M. Odelius, Phys. Rev. Lett. 82, 3919 (1999).

[17] Y. Ohta, T. Haga, and Y. Abe, Jpn. J. Appl. Phys. 36, L1040 (1997). 